\title{
Atitudes e Conhecimentos de PROFissionais de SAÚde diante do ÁlCool, ALCOOLISMO E DO ALCOOLISTA: LEVANTAMENTO DA PRODUÇÃo CIENTÍFICA NOS ÚLTIMOS 50 ANOS
}

\section{Janaina Soares ${ }^{1}$; Divane de Vargas ${ }^{2}$; Márcia Aparecida Ferreira de Oliveira ${ }^{3}$}

O estudo verificou a produção científica sobre a temática atitudes e conhecimentos de profissionais de saúde diante do álcool, do alcoolismo e do alcoolista, nos últimos 50 anos, por meio de consulta sistemática às bases de dados: PubMed, LILACS e SciELO. Procedeu-se à análise do conteúdo que originou três temáticas. Os resultados evidenciaram que a maioria dos profissionais apresentou atitudes que não se diferenciam daquelas encontradas no senso comum, percebendo-se que as atitudes dos profissionais de saúde sotreram poucas mudanças ao longo das últimas cinco décadas.

Descritores: Atitude do Pessoal de Saúde; Conhecimentos, Atitudes e Prática em Saúde; Alcoolismo.

\section{Health Professionals' attitudes and knowledge about alcohol, ALCOHOLISM AND ALCOHOLICS: A SURVEY OF THE SCIENTIFIC PUBLICATIONS IN THE LAST 50 YEARS}

This study verified the scientific publications on the theme health professionals' attitudes and knowledge about alcohol, alcoholism and alcoholics in the last 50 years through systematic searches on the following databases: PUBMED, LILACS and SCIELO. The content analysis resulted in three thematic groups. Results evinced that most professionals showed attitudes indifferent from those of common sense. Thus, it was concluded that the attitudes of health professionals have not suffered many changes over the last five decades.

Descriptors: Attitude of Health Personnel; Health Knowledge, Attitudes, Practice; Alcoholism.

\footnotetext{
1-Enfermeira, Mestranda em Enfermagem, Escola de Enfermagem, Universidade de São Paulo, SP, Brasil. E-mail: janainasoares@usp.br 2-Enfermetro, Doutor em Enfermagem, Professor Doutor, Departamento de Enfermagem Materno-Infantil e Psiquiátrica, Escola de Enfermagem, Universidade de São Paulo, SP, Brasil. E-mail: vargas@usp.br

3-Enfermeira, Doutor em Ciências Sociais, Professor Associado, Departamento de Enfermagem Materno-Infantil e Psiquiátrica, Escola de Enfermagem, Universidade de São Paulo, SP, Brasil.

Endereço para Correspondência:

Divane de Vargas

Universidade de São Paulo

Escola de Enfermagem

Departamento de Enfermagem Materno Infantil e Psiquiátrica

Avenida Dr. Enéas de Carvalho Aguiar, 419

CEP 05403-000

São Paulo, SP, Brasil

E-mail: vargas@usp.br
} 
ACTITUdes Y CONOCIMIENTO DE PROFESIONALES DE LA SALUd FRENTE AL ALCOHOL, AL ALCOHOLISMO Y AL ALCOHÓLICO: REVISIÓN DE LA PRODUCCIÓN CIENTÍFICA DE LOS ÚlTIMOS 50 AÑOS

El estudio verificó la producción científica sobre la temática de actitudes y conocimientos de profesionales de la salud frente al alcohol, al alcoholismo y al alcohólico en los últimos 50 años a través de consulta sistemática a las bases de datos: PUBMED, LILACS y SCIELO. Se procedió a un análisis del contenido que dio origen a tres temáticas. Los resultados evidenciaron que la mayoría de los profesionales presentó actitudes que no se diferencian de aquellas encontradas en el sentido común, percibiéndose que las actitudes de los profesionales de la salud sufrieron pocos cambios a lo largo de las últimas cinco décadas.

Descriptores: Actitud del Personal de Salud; Conocimientos Actitudes y Práctica en Salud; Alcoholismo. 


\section{Introdução}

O álcool é consumido pelo homem desde os tempos mais remotos, presente em diversas culturas, e lembrado que, biblicamente, era relacionado ao sexo e à luxúria, e, por muitos anos, o alcoolismo representou fraqueza de caráter daqueles que o consumiam ${ }^{(1)}$. Atualmente, apesar de sua ampla aceitação social ${ }^{(2)}$, o consumo de álcool em excesso é considerado um dos problemas médicosociais mais graves da sociedade moderna, pois, o mesmo tem gerado altos custos para sociedade, o que pode ser constatado pelos relatos científicos de prejuízos físicos, psicológicos e outros distúrbios sociais, atribuídos ao mesmo $^{(1,3-4)}$, sendo consistente o fato de que o consumo de álcool está associado a mais de 60 patologias relacionadas na CID $10^{(5)}$.

Segundo o Relatório sobre a Saúde no Mundo ${ }^{(6)}$, o álcool representa $3,2 \%$ de todas as mortes no mundo, constituindo-se em um dos principais riscos à saúde nos países em desenvolvimento, com baixa mortalidade. Nos Estados Unidos, o problema atinge cerca de 5 a 10\% das pessoas $^{(7)}$, na América Latina de 3 a $23 \%$ da população é acometida pela dependência do álcool ${ }^{(8)}$. No Brasil, um levantamento nacional ${ }^{(9)}$ evidenciou que $12,3 \%$ das pessoas, com idade entre 12 e 65 anos, são dependentes de álcool.

Esses prejuízos têm acarretado, dentre outros, aumento da demanda de pessoas com problemas relacionados ao uso/ abuso de álcool e outras drogas nos serviços de saúde ${ }^{(2,8)}$. Fato que tem cada vez mais contribuído para aumentar o contato dos profissionais de saúde com essa população. Entretanto, segundo o Ministério da Saúde ${ }^{(10)}$, apesar desse aumento na demanda de pessoas com problemas relacionados ao álcool e ao alcoolismo nos serviços de atenção à saúde, existem diversos impedimentos para diagnosticar, tratar ou encaminhar as pessoas que apresentam complicações decorrentes do consumo de álcool. Em um plano cognitivo, os trabalhadores de saúde apresentam a falta de conhecimentos sobre a variedade de apresentações sintomáticas, geradas pelo uso abusivo e pela dependência do álcool, bem como de meios para facilitar o seu diagnóstico. Apresentam, também, visão negativa do paciente e de suas perspectivas evolutivas diante do problema, o que impede atitude mais produtiva. Essa constatação, no entanto, não se restringe somente ao contexto brasileiro, existindo evidências de que se trata de problemática mundial ${ }^{(11)}$.

Apesar dessas evidências, o tema atitudes e conhecimentos dos profissionais da saúde diante do álcool e outras drogas tem despertado pouco interesse dos pesquisadores, de um modo geral ${ }^{(2,12)}$, e, apesar de estudos realizados nos Estados Unidos e outros países da Europa, no Brasil essa temática começou a ser explorada a partir da segunda metade da década de 1990, o que causou, até então, lacuna nessa área do conhecimento. Diante disso, torna-se oportuno realizar estudo com o objetivo de identificar e analisar as produções científicas sobre a temática atitudes e conhecimentos de profissionais de saúde diante do álcool, alcoolismo e do alcoolista nos últimos 50 anos.

\section{Metodologia}

Este é um estudo exploratório de cunho bibliográfico, o qual utilizou, para a realização da pesquisa, consulta sistemática às bases de dados: PubMed, LILACS e SciELO, estipulando-se o período correspondente aos anos de 1959 a 2009. Para coleta dos dados, elegeramse os seguintes descritores: 1. attitudes, 2. knowledge, 3. alcohol e 4. alcoholism. Foram encontrados 349 artigos, no total, dos quais apenas 16 artigos preencheram os critérios de inclusão na amostra. Como critérios de inclusão para a seleção dos estudos, consideraram-se os estudos que contivessem os descritores (utilizados juntos nas línguas portuguesa e inglesa), abordassem a temática em estudo, terem sido publicados entre 1959 e 2009 e estarem disponíveis na íntegra nas bases investigadas. Como critérios de exclusão, consideraram-se os artigos que não preencheram os requisitos citados acima, artigos que exploravam as atitudes e conhecimentos de apenas um grupo de profissionais e que abordassem outras substâncias psicoativas.

Em seguida, os artigos foram distribuídos por décadas de publicação, durante o período de 1959 a 2009 , e quanto aos países de publicação. Concluída essa fase, iniciou-se o processo de classificação dos artigos quanto às atitudes e conhecimentos de profissionais de saúde durante as décadas. Essa parte da pesquisa exigiu abordagem qualitativa, na qual se utilizou a análise de conteúdo, que trata de um conjunto de técnicas de análise de textos ou comunicações, visando obter, através de procedimentos sistemáticos e objetivos da descrição de seus conteúdos, indicadores quantitativos ou não, que permitam inferir conhecimentos relativos às condições de produção deduzidas desses textos ${ }^{(13)}$. Para categorização do material, esse tratamento dos dados se caracterizou pela leitura exaustiva das publicações, procurando determinar como as atitudes e conhecimentos diante do álcool, alcoolismo e do alcoolista têm sido abordadas nos últimos cinquenta anos.

A análise de conteúdo dos dados coletados permitiu a categorização, a qual se refere ao agrupamento de elementos com características comuns, relacionadas entre si, ou seja, esse procedimento de categorização representa o agrupamento de ideias ou expressões em torno de um conceito comum, capaz de abranger todo o conjunto de dados $^{(14)}$, os quais originaram a categoria Atitudes e conhecimentos de profissionais de saúde diante do álcool, alcoolismo e do alcoolista, subdividida em três temáticas para a análise: I. atitudes de profissionais da saúde, II. conhecimento de profissionais da saúde e III. atitudes e conhecimentos.

\section{Resultados}

A busca sistemática nos bancos de dados resultou numa amostra de 16 artigos. Analisando a distribuição das publicações, nas cinco décadas investigadas, observa-se que a maior concentração $(37,5 \%)$ de publicações se deu no período de 2000 a 2009 (Figura 1). 


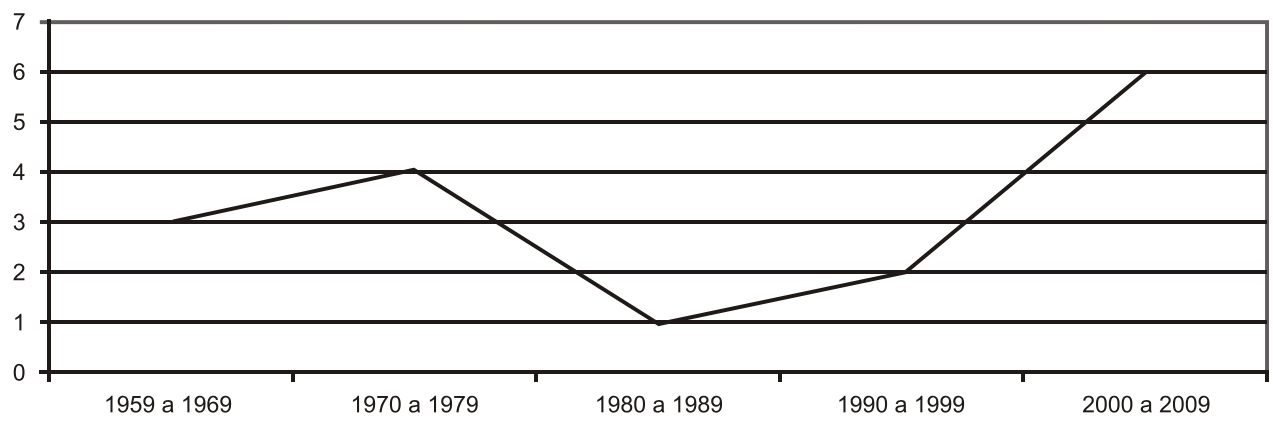

Figura 1 - Distribuição dos artigos $(n=16)$ sobre as atitudes e conhecimentos dos profissionais de saúde diante do alcool, alcoolismo e do alcoolista, entre 1950 e 2009

Com relação à categoria profissional, os enfermeiros constituem o grupo que tem despertado maior interesse dos pesquisadores, no que se refere às atitudes diante do álcool e do alcoolismo, e estudos, envolvendo esses profissionais, correspondem a $52,2 \%$ do total. A origem dessas publicações, segundo a amostra analisada, é predominantemente da Inglaterra $(56,25 \%)$, seguida dos EUA (18,75\%) (Tabela 1).

Tabela 1 - Distribuição do número de artigos sobre as atitudes e conhecimentos dos profissionais de saúde diante do álcool, alcoolismo e do alcoolista, durante as décadas, de acordo com os países estudados

\begin{tabular}{lcc}
\hline \multicolumn{1}{c}{ Países } & Total & $\%$ \\
\hline Austrália & 2 & 12,5 \\
Inglaterra & 9 & 56,25 \\
EUA & 3 & 18,75 \\
Canadá & 1 & 6,25 \\
Irlanda & 1 & 6,25 \\
Total & 16 & 100 \\
\hline
\end{tabular}

A análise qualitativa das publicações permitiu, conforme mencionado, o agrupamento dos dados em uma categoria a qual foi subdividida em três temáticas, descritas na sequência.

CATEGORIA - Atitudes e conhecimentos de profissionais da saúde diante do álcool, do alcoolismo e do alcoolista

Temática I. Atitudes de profissionais da saúde

Esta temática refere-se aos estudos sobre as atitudes de profissionais de saúde diante do álcool, alcoolismo e do alcoolista, e foi composta por seis publicações, as quais passarão a ser descritas resumidamente, na sequência.

Em um estudo ${ }^{(15)}$, avaliando as atitudes de enfermeiros que trabalhavam em sanatórios de tuberculosos, sobre pacientes com duplo diagnóstico de tuberculose e alcoolismo, encontrou-se que os enfermeiros apresentavam atitudes positivas diante do tratamento do alcoolismo para esses pacientes.

Nessa pesquisa ${ }^{(16)}$, verificaram as atitudes e crenças de profissionais dos setores de saúde diante de pacientes alcoolistas, dentre eles médicos, enfermeiros e assistentes sociais, e encontrou-se que metade dos participantes concordaram ser importante a vontade do paciente para o sucesso do tratamento. Os profissionais com atitudes moralistas diante de alcoolistas estavam significativamente mais motivados a fazer julgamento à motivação do alcoolista ao tratamento do que os profissionais não moralistas, e, ainda, mostrou que enfermeiros tiveram atitudes mais moralistas diante de alcoolistas do que os assistentes sociais e os médicos.

Estudo $^{(17)}$ sobre as atitudes diante de alcoolistas de membros dos Alcoólicos Anônimos, pacientes alcoolistas hospitalizados, pacientes alcoolistas ambulatoriais e profissionais que trabalham e que não trabalham com alcoolistas verificou que os membros dos Alcoólicos Anônimos eram mais otimistas que os participantes dos outros grupos, entretanto, profissionais e equipes que trabalham com pacientes alcoolistas eram menos pessimistas e concebem o alcoolismo como doença.

$\mathrm{Na}$ década de $70^{(18)}$, enviaram um questionário a médicos e enfermeiros do Sul da Austrália, com o objetivo de verificar as atitudes desses profissionais diante do álcool e do alcoolismo. Dentre $33 \%$ dos médicos e enfermeiros que retornaram o questionário, um quinto dos médicos e mais de um terço dos enfermeiros concordaram que o alcoolismo é um problema moral ou espiritual, e, ainda, dentre os enfermeiros, consideraram a personalidade do indivíduo como a mais relevante causa do alcoolismo.

Nessa mesma década ${ }^{(19)}$, verificaram as atitudes em relação ao alcoolismo entre psiquiatras, psicólogos, assistentes sociais, enfermeiros e auxiliares de enfermagem, através de um questionário. Entre a amostra estudada, foi predominante a associação de dificuldades emocionais com o alcoolismo, atitudes positivas em relação à recuperação e tratamento, a associação da ingestão de bebidas alcoólicas em excesso com sua dependência e, além disso, os profissionais não consideraram o alcoolista como indivíduo com problemas de caráter. O grupo de psicólogos desconsiderou os conceitos de doença e de responsabilidade em relação ao vício. Auxiliares de enfermagem definiram o alcoolista como um bebedor pesado e pessoa inofensiva.

Outro estudo ${ }^{(20)}$, no qual foi proposto analisar os hábitos de beber pessoais, atitudes sobre o beber e preferência de tratamento, sugerido para alcoolistas de uma equipe de trabalho de um centro médico, verificou- 
se que não houve diferenças entre as atitudes de médicos, psicólogos e assistentes sociais, embora apresentassem mais atitudes positivas diante do beber social e menos atitudes punitivas em comparação aos enfermeiros. Dentre os enfermeiros, aqueles que relataram moderado consumo de álcool apresentaram maior aceitação diante do beber social e menos atitudes punitivas com relação ao alcoolista do que aqueles enfermeiros que não consumiam álcool.

Temática II. Conhecimentos de profissionais da saúde

Esta temática refere-se às publicações que abordam estudos relacionados ao conhecimento de profissionais de saúde diante do álcool, do alcoolismo e do alcoolista. Essa temática é composta por quatro artigos descritos em seguida.

Pesquisa $^{(21)}$, na qual se avaliaram os cuidados hospitalares para o doente, mostrou que a maioria dos médicos e enfermeiros referiram não encontrar pacientes com problemas de uso de álcool relevantes ou histórias de uso de drogas, não identificar a dependência química como um problema médico, mesmo quando identificada, além de não se envolverem em recomendações de tratamento. Já(22), avaliando o nível de conhecimento sobre o álcool dos profissionais médicos, enfermeiros e policiais do Nordeste da Inglaterra, descobriu-se que os médicos não tinham conhecimento sobre limites de consumo de álcool, e mais de um terço da amostra de enfermeiros mostrou não saber que esses limites são menores para as mulheres.

Em outro estudo ${ }^{(23)}$, visando identificar as barreiras para os prestadores de cuidados de saúde na realização de intervenções breves para bebedores pesados, utilizouse um questionário sobre as atitudes, habilidades, conhecimentos, necessidades de formação e sugestões para implementação de intervenções breves, que foi enviado a todos os enfermeiros e médicos que trabalhavam nos cuidados primários de saúde, em duas cidades finlandesas. Os resultados apontaram que os fatores relacionados ao conhecimento pareceram ser uma barreira para a adoção da intervenção breve, pois, apenas $18 \%$ dos entrevistados afirmaram ter conhecimento suficiente para fornecer intervenção breve, competentemente, e a outra metade dos entrevistados disse que necessitava de mais formação. Contrariamente às expectativas, os médicos consideram-se mais bem preparados para realizar intervenções breves do que os enfermeiros.

Realizando um estudo ${ }^{(24)}$ com o objetivo de investigar diferenças entre os conhecimentos de profissionais da saúde de unidades de atendimento de saúde rurais e urbanas, para sensibilização à síndrome fetal do álcool (SAF) e ao aconselhamento pré-concepção, enviaram-se inquéritos a profissionais de saúde canadenses e foi verificado que algumas diferenças entre os profissionais de saúde rurais e urbanos existiam, no que diz respeito ao conhecimento e ao diagnóstico da SAF, no entanto, os profissionais da saúde de unidades rurais estavam mais preparados para cuidar de mulheres com problemas de dependência e eram mais propensos a cuidar de pacientes com SAF.
Temática III. Atitudes e conhecimentos

Esta temática refere-se a estudos que abordaram especificamente a relação entre atitudes e conhecimentos de profissionais da saúde dos diversos espaços de atuação (unidades básicas de saúde, hospitais, clínicas especializadas, empresas etc.) diante de questões relacionadas ao uso de bebidas alcoólicas, ao beber social, ao alcoolista, tratamentos e à etiologia do alcoolismo. Compõem essa temática seis artigos, relacionados ao tema atitudes e conhecimentos, a metade dos artigos compara médicos e enfermeiros em relação às atitudes diante do usuário de álcool.

Pesquisa $^{(25)}$ sobre os conhecimentos, atitudes e crenças, relacionadas ao álcool, de médicos, enfermeiros e executivos mostrou que os médicos tiveram atitudes mais permissivas em relação ao uso de álcool em diferentes situações sociais e eram menos propensos a recomendar o tratamento obrigatório do que os enfermeiros ou executivos. No que se refere à influência para o uso de bebidas, os enfermeiros e executivos consideraram o aumento do preço das bebidas alcoólicas, uma abordagem menos eficaz, enquanto os médicos consideraram a imprensa como fonte menos influente.

Em outro estudo ${ }^{(26)}$ sobre as atitudes dos médicos de família canadenses, crenças e práticas em matéria de uso de álcool e problemas relacionados ao álcool, entre seus pacientes, a partir de uma entrevista, verificou-se que os entrevistados achavam relevante a atenção aos alcoolistas, mas mostraram atitudes predominantemente negativas e pessimistas em relação ao mesmo. Metade dos entrevistados mostrou nunca ter tido contato com alcoolistas. Os médicos conceberam o alcoolismo como etiologia psicossocial. Três quartos dos entrevistados disseram "quase sempre" perguntar aos pacientes sobre a sua frequência do uso de álcool. Os dados também sugerem que os médicos identificaram poucos pacientes com problemas de álcool, e que precisam de ajuda para identificar tais pacientes.

Outra pesquisa ${ }^{(27)}$ sobre as atitudes e práticas dos médicos de clínica geral e enfermeiros, sobre a identificação precoce e intervenção para problemas relacionados ao álcool, mostrou que a abordagem sobre o uso de álcool foi maior entre os médicos do que entre enfermeiros. Ambos os grupos abordam o consumo de álcool nos casos em que eles acreditavam que o estado de saúde foi influenciado pelo álcool. Para ambas as profissões, conhecimentos e competências em matéria de identificação foram mais bem compreendidos no que se refere aos métodos de intervenção. Enfermeiros, avaliando os seus conhecimentos e habilidades, apresentaram-se menos confiantes do que os médicos. A atitude geral foi bastante positiva para a identificação e intervenção precoce, mas enfermeiros estavam mais preocupados do que os médicos em relação ao fato de os pacientes reagirem negativamente ao rastreamento sobre o uso de álcool

Em estudo(28), investigando as atitudes dos profissionais de saúde mental à droga e toxicodependência, 
em Victoria, Austrália, aplicou-se um questionário sobre conhecimentos, habilidades, atitudes e práticas que foi distribuído aos médicos de saúde mental. Os resultados da pesquisa mostraram que a atitude dos profissionais de saúde mental para drogas e abuso de substâncias é geralmente positiva. A maioria dos entrevistados tinha opiniões positivas sobre as intervenções de tratamento e se mostraram otimistas quanto à dependência de drogas e álcool ser doença tratável.

Numa avaliação ${ }^{(29)}$ referente à relação às mudanças de atitudes, habilidades e conhecimento sobre a intervenção breve, entre enfermeiros e médicos de clínica geral na atenção primária à saúde, na cidade de Tampere, Finlândia, através de um questionário, verificaram-se várias mudanças positivas indicando aumento do conhecimento a respeito de intervenção breve entre os profissionais, durante a execução, principalmente entre os enfermeiros.

No estudo $^{(30)}$ sobre as atitudes e crenças dos funcionários do departamento da emergência de um hospital de Sydney, em relação ao álcool, em uma amostra de $54 \%$ enfermeiros e $46 \%$ de médicos, identificouse que apenas 5\% geralmente realizava formalmente o rastreamento de problemas de álcool, apenas $16 \%$ geralmente realizava intervenções breves, e apenas $27 \%$ geralmente proporcionava encaminhamento para os serviços de tratamento especializado. Mais de $85 \%$ dos profissionais indicaram que as dificuldades para a realização de intervenções estão relacionadas à falta de motivação do paciente.

\section{Discussão}

A análise realizada, a partir dos resultados encontrados no levantamento das produções de profissionais de saúde diante do álcool, alcoolismo e do alcoolista, nos últimos cinquenta anos, possibilitou constatar que, embora ainda se apresentem em número bastante reduzido, quando comparadas com outras temáticas sobre o assunto, houve aumento das produções no período de 2000 a 2009, em relação aos outros períodos.

No que se refere à procedência desses estudos, a maioria foi publicada na Inglaterra $(56,25 \%)$, seguido dos EUA $(18,75)$, apesar de o Brasil ter contribuído significativamente com estudos avaliando atitudes e conhecimentos de enfermeiros ${ }^{(31-33)}$, o mesmo não se observa no estudo de outras categorias profissionais, o que abre espaço para pesquisas nessa área. De acordo com os profissionais estudados, predominaram estudos sobre as atitudes e conhecimentos de enfermeiros seguido de profissionais médicos.

Com relação ao cenário de recrutamento dos profissionais de saúde para pesquisa de atitudes e conhecimentos diante do álcool, alcoolismo e do alcoolista, predominam os estudos realizados com enfermeiros de unidades de saúde e serviços ${ }^{(20,28)}$ de atenção primária ${ }^{(23-}$ 24,26-27,29), compondo, assim, populações investigadas com relação às atitudes e conhecimentos de profissionais de unidades hospitalares ${ }^{(15,17,21,30)}$, dentre outros.

Segundo as publicações referentes a atitudes pessoais dos profissionais de saúde diante do álcool, alcoolismo e do alcoolista, destacaram-se ${ }^{(15-20)}$ os estudos sobre os conhecimentos desses profissionais ${ }^{(21-24)}$, discutiram-se as questões relacionadas à identificação do alcoolista, à etiologia do alcoolismo, além de artigos que se ocupam de ambos os assuntos (atitudes e conhecimentos) ${ }^{(25-30)}$, geralmente comparando a influência de um no outro, ou seja, se quanto maior o conhecimento há mais atitudes positivas $^{(28-30)}$.

Os dados ${ }^{(20)}$ sobre atitudes e conhecimentos de profissionais diante do álcool, alcoolismo e do alcoolista permitiram evidenciar que, em relação ao álcool, os médicos consideraram o beber social atitudes mais positivas e menos punitivas que os enfermeiros, entretanto, enfermeiros que fazem uso moderado de bebida alcoólica apresentaram maior aceitação ao beber social e menos atitudes punitivas do que enfermeiros que não fazem o consumo de bebidas alcoólicas. Esse fato pode estar relacionado à visão dominante da sociedade que aceita o beber moderado e rejeita o indivíduo que bebe descontroladamente ${ }^{(31)}$, e, ainda, pode estar correlacionado às concepções e vivências proporcionadas por experiências desses profissionais ao prestarem assistência a pacientes com problemas decorrentes do beber ${ }^{(34)}$.

Já no que se refere às atitudes sobre o alcoolismo, os profissionais que trabalham com pacientes alcoolistas ou profissionais da área de saúde mental, concebem o alcoolismo como doença ${ }^{(17,28)}$, porém, em outros estudos $^{(19,25)}$, psicólogos tenderam a negar o conceito de doença ao alcoolismo e médicos e enfermeiros ${ }^{(18-19)}$ consideraram o alcoolismo como problema moral ou espiritual, além de o relacionar às dificuldades emocionais e à personalidade como causas mais relevantes. Estudo ${ }^{(35)}$ sobre atitudes de profissionais para com o alcoolismo sugere a falta de aceitação do mesmo, sendo que vários grupos de profissionais reconhecem o alcoolismo intelectualmente, refletindo na questão de ser mais fácil aceitar isso intelectual do que emocionalmente.

Analisando as atitudes diante do alcoolista, os profissionais tenderam a mostrar opiniões moralistas ${ }^{(17)}$, julgando a motivação do mesmo ao tratamento ${ }^{(15,17,26)}$, porém, verificou-se $\mathrm{e}^{(18)}$ que os profissionais de saúde que trabalham ou que têm contato maior com pacientes alcoolistas são menos pessimistas ao tratamento, acreditam na recuperação do paciente e não acham que esses pacientes possuem deficiência de caráter ${ }^{(19)}$.

A análise das publicações sobre o conhecimento permitiu verificar que, de maneira geral, para esses profissionais foi evidente a dificuldade em lidar com esses indivíduos devido à falta de conhecimento suficiente sobre as formas de abordagem ${ }^{(21)}$, como identificação precoce, aconselhamento, intervenções, encaminhamento para especialidades, limites do consumo e suas diferenças entre os gêneros ${ }^{(22)}$, tornando-se a falta de conhecimento barreira à aderência desses profissionais para realizarem as intervenções competentemente ${ }^{(26)}$, correspondendo ao interesse dos mesmos em maior formação na temática como suporte para a detecção desses problemas ${ }^{(23)}$, o que, segundo os autores ${ }^{(36)}$, pode ser atribuído ao fato de 
esses profissionais terem recebido pouca informação e treinamento específico sobre o tema, refletindo, ao mesmo tempo, a pouca atenção dada à temática na formação dos profissionais de saúde, tornando o conhecimento insuficiente.

Diante disso, pode-se conceber que as atitudes diante do álcool, do alcoolismo e do alcoolista podem estar diretamente relacionadas à falta de conhecimento do profissional, considerando que, conforme o profissional pratica esse cuidado, consegue adquirir cada vez mais habilidades, assim como melhorar a segurança e apresentar atitudes mais positivas diante do cuidado ao usuário de álcool ${ }^{(27,29)}$, contudo, aumentar as competências motivacionais à prática, em especial, parece ser desafio futuro $^{(29)}$.

\section{Considerações Finais}

Existe número reduzido de publicações sobre atitudes e conhecimentos de profissionais de saúde diante do álcool, alcoolismo e do alcoolista, evidenciando que o tema tem despertado pouco interesse entre os pesquisadores sobre a temática em todo o mundo.

Os resultados permitiram evidenciar que a falta de conhecimentos pode ser atribuída às atitudes negativas, pois profissionais que mostraram ter maior contato com esses usuários tenderam, consequentemente, a apresentar mais habilidades do que aqueles que tiveram pouco ou nenhum contato com esses pacientes, além de mostrarem atitudes mais positivas e melhor conhecimento sobre a temática, assim, a prevalência de atitudes negativas pode estar relacionada ao pouco interesse do ensino, durante a formação desses profissionais, e por não terem tido contato com essa problemática.

Existe a necessidade de maior atenção a esse tema, uma vez que as atitudes e conhecimentos influenciam diretamente na qualidade de atenção dos profissionais de saúde, em geral, para com as pessoas que sofrem de problemas relacionados ao álcool e ao alcoolismo, além disso, todos os profissionais de saúde devem estar preparados para atender essa demanda, uma vez que se trata de problema de saúde pública mundial.

\section{Referencias}

1. Vargas D. A construção de uma escala de atitudes frente ao álcool, ao alcoolismo e ao alcoolista: um estudo psicométrico. [Tese]. Ribeirão Preto: Escola de Enfermagem de Ribeirão Preto, Universidade de São Paulo; 2005.

2. Vargas D, Luis MAV. Álcool, alcoolismo e alcoolista: concepções e atitudes de enfermeiros de unidades básicas distritais de saúde. Rev. Latino-Am. Enfermagem. 2008;16 n.spe:543-50.

3. Lucca DM, Vargas D, Vargas D. As concepções de enfermeiros de hospital geral frente as questões relacionadas ao álcool e ao alcoolismo. Rev Enferm Bras. 2006;5(5):260-6.

4. Navarrete PR, Luís MAV. Atitude da enfermeira de um complexo hospitalar em relação ao paciente alcoólico. Rev. Latino-Am. Enfermagem. 2004;12:420-6.

5. Ministério da Saúde (BR). Secretaria Nacional Antidrogas. Glossário de álcool e drogas. Brasília; 2006.

6. Organização Mundial de Saúde. Segundo Relatório sobre saúde no mundo. 2002. [página na Internet] 2007. [acesso 12 fev 2010] Disponível em: http://www.who.int/ whr/2001/en/whr01_djmessage_po.pdf.

7. Lewis DC. The role of the generalist in the care of substance abusing patient. Med Clin North Am. 1997;81:831-43.

8. Vargas D, Araújo E. Prevalência de dependência alcoólica em serviços de atenção primária a saúde de Bebedouro (SP) - Brasil. Cad Saúde Pública. 2009;25(8):1711-20.

9. Carlini EA, Galduróz JCF, Noto AR, Nappo SA. II Levantamento domiciliar sobre uso de drogas psicotrópicas no Brasil - 2005. São Paulo: Centro Brasileiro de Informações sobre Drogas Psicotrópicas/Departamento de Psicobiologia, Universidade Federal de São Paulo; 2006. 10. Ministério da Saúde (BR). Secretaria de Atenção à Saúde. SVS/CN-DST/AIDS. A Política do Ministério da Saúde para Atenção Integral a Usuários de Álcool e outras Drogas/Ministério da Saúde. Brasília; 2004.

11vGriffiths RD, Stone A, Tran DT, Fernandez RS, Ford K. Drink a little; take a few drugs: do nurses have knowledge to identify and manage in-patients at risk of drugs and alcohol? Drug Alcohol Rev. 2007;26(5):545-52.

12. Howard MO, Chung SS. Nurses' attitudes toward substance misusers I. Surveys, Subst Use and Misuse. 2000;35(3):347-65.

13. Bardin L. Análise de conteúdo. Lisboa: Edições 70; 1979.

14. Deslandes SF. Pesquisa Social: teoria, método e criatividade. Petropolis (RJ): Vozes; 1994.

15. Linsky AS, Heinemann ME, Sorensen KM. Problems of the alcoholic-tuberculous patient. A study of attitudes of the hospitalized alcoholic-tuberculous, and the nurse. Nurs Res. 1965;14:33-6.

16. Sterne MW, Pittman DJ. The concept of motivation: A source of institutional and professional blockage in the treatment of alcoholics. Q J Stud Alcohol. 1965;26:41-57. 17. Mogar RE, Helm ST, Snedeker MR, Snedeker MH, Wilson WM. Staff attitudes toward the alcoholic patient. Arch Gen Psychiatry. 1969;21(4):449-54.

18. Van B, Gabrynowicz JW. Doctors' and nurses' views on alcohol dependency. Aust Nurses J 1977;7(4):42-5.

19. Lemos AV, Moran J. Veterans Administration hospital staff attitudes toward alcoholism. Drug Alcohol Depend. 1978; 3(2):77-88.

20. McLellan AT, Hery DS, Druley KA. Staff drinking patterns and approach to patient drinking problems within a psychiatric hospital. Am J Drug Alcohol Abuse. 1978;5(4):507.

21. Westermeyer J, Doheny S, Stone B. An assessment of hospital care for the alcoholic patient. Alcohol Clin Exp Res. 1978;2(1):53-7.

22. Myszor M, Hosker H, Foster H, Record C. Alcohol and health: do we know enough? Health Trends. 1990;22(1):23-7. 
23. Aalto M, Pekuri P, Seppä K. Primary health care nurses' and physicians' attitudes, knowledge and beliefs regarding brief intervention for heavy drinkers. Addiction. 2001;96(2):305-11.

24. Tough SC, Ediger K, Hicks M, Clarke M. Rural-urban differences in provider practice related to preconception counselling and fetal alcohol spectrum disorders. Can J Rural Med. 2008;13(4):180-8.

25. Poikolainen K. Alcohol-related knowledge, beliefs and attitudes among health and clerical personnel. Soc Sci Med. 1988;27(12):1429-32.

26. Rush B, Bass M, Stewart M, McCracken E, Labreque M, Bondy S. Detecting, preventing, and managing patients' alcohol problems. Can Fam Physician. 1994;40:1557-66. 27. Johansson K, Bendtsen P, Akerlind I. Early intervention for problem drinkers: readiness to participate among general practitioners and nurses in Swedish primary health care. Alcohol Alcohol. 2002;37(1):38-42.

28. Pinikahana J, Happell B, Carta B. Mental health professionals' attitudes to drugs and substance abuse. Nurs Health Sci. 2002;4(3):57-62.

29. Aalto M, Pekuri P, Seppä K. Implementation of brief alcohol intervention in primary health care: do nurses' and general practitioners' attitudes, skills and knowledge change? Drug Alcohol Rev. 2005;24(6):555-8.

30. Indig D, Copeland J, Conigrave KM, Rotenko I. Attitudes and beliefs of emergency department staff regarding alcohol-related presentations. Int Emerg Nurs. 2009;17(1):23-30.

31. Pillon CS, Luís MAV, Laranjeira R. Atitudes dos enfermeiros relacionadas ao alcoolismo. Rev Nurs. 2006; 96(9):811-6.

32. Lucca DM, Vargas D, Vargas D. As concepções de enfermeiros de hospital geral frente as questões relacionadas ao álcool e ao alcoolismo. Rev Enferm Bras. 2006;5(5):260-6.

33. Vargas D, Labate RC. Atitudes de enfermeiros de hospital geral frente ao uso do álcool e alcoolismo. Rev. Bras. Enferm. 2006;59(1):47-51.

34. Allen K. Attitudes of registered nurses toward alcoholic patients in a general hospital population. The International Journal of the Addictions. 1993;28(9):923-30.

35. Vargas HS. Repercussões do álcool e do alcoolismo. São Paulo (SP) Byk-Procienx; 1983.

36. Pillon SC, Laranjeira RR. Formal education and nurses' attitudes towards alcohol and alcoholism in a Brazilian sample. São Paulo Med J. 2005;123(4):175-80.

\section{Como citar este artigo:}

Soares J, Vargas D, Oliveira MAF. Atitudes e conhecimentos de profissionais de saúde diante do álcool, alcoolismo e do alcoolista: levantamento da produção científica nos últimos 50 anos. SMAD, Rev. Eletrônica Saúde Mental Álcool Drog. (Ed. port.). jan-abr 2011 [acesso:

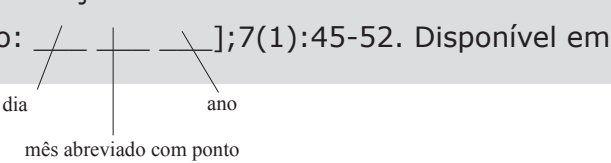

